Breakdown Performance

\title{
Statistics of a Nanoparticle Composite System
}

D. Sanders, E. Cook, R. Anaya, L. Wang, S.

Sampayan, G. Caporaso, K. Slenes, J. Jacquin, R. De La Fuenta

July 26, 2007

2007 IEEE Pulsed Power Conference and Plasma Science Conference

Albuquerque, NM, United States

June 17, 2007 through June 22, 2007 
This document was prepared as an account of work sponsored by an agency of the United States government. Neither the United States government nor Lawrence Livermore National Security, LLC, nor any of their employees makes any warranty, expressed or implied, or assumes any legal liability or responsibility for the accuracy, completeness, or usefulness of any information, apparatus, product, or process disclosed, or represents that its use would not infringe privately owned rights. Reference herein to any specific commercial product, process, or service by trade name, trademark, manufacturer, or otherwise does not necessarily constitute or imply its endorsement, recommendation, or favoring by the United States government or Lawrence Livermore National Security, LLC. The views and opinions of authors expressed herein do not necessarily state or reflect those of the United States government or Lawrence Livermore National Security, LLC, and shall not be used for advertising or product endorsement purposes. 


\title{
Breakdown Performance Statistics of a Nanoparticle Composite System
}

\author{
Dave Sanders, Ed Cook, Rick Anaya, Lisa Wang, Steve Sampayan and George Caporaso \\ Lawrence Livermore Lab, Livermore, CA, USA
}

Kirk Slenes, Jeff Jacquin and Rafael De La Fuente

TPL Inc, Albuquerque, NM, USA

\begin{abstract}
The Dielectric Wall Accelerator (DWA) is an approach for manufacturing particle accelerators that can be an order of magnitude more compact than conventional accelerators. To realize the associated high level of electric field gradient it is necessary to develop and characterize materials that can maintain electric fields in excess of $100 \mathrm{MV} / \mathrm{m}(=1 \mathrm{MV} / \mathrm{cm})$. This high value for the breakdown strength must be maintained for many thousands of pulses under conditions of voltage reversal. The present work reports the performance of a candidate composite material composed of high dielectric constant nanoparticles dispersed in an epoxy matrix loaded to achieve a dielectric constant $=$ 10. The effect of voltage on number of pulses that can be sustained is given. In addition the effects of electrode gap spacing and charging rate are explored.
\end{abstract}

\section{INTRODUCTION}

Electron accelerators can be are used make high energy x-rays for tomography, x-ray based cancer treatment as well as for such diverse applications as medical diagnostics and baggage inspection. Particle accelerators can also be used to accelerate protons used for cancer therapy. Deuterium accelerators can be used to produce neutrons which, in turn, can be used in the detection of explosives and special nuclear material. All of these applications will benefit from the existence of more compact accelerators that are made possible with DWA designs. One of the determining factors in a DWA's compactness is the voltage holding ability of the materials used in their construction. In the present work a candidate material system formulated as a suspension of high dielectric nanoparticles suspended in an epoxy matrix with a dielectric constant $=10$ is evaluated. Since the insulating materials in a DWA will be subject to many thousands of pulses with voltage reversal, we decided to construct equipment that could subject the material under study to similar fields and voltage reversals for multiple pulses in an automated system.

\section{EXPERIMENTAL}

Figure 1a shows a schematic for the circuit model. Capacitors C1 and C2 and inductors L1 and L2 model the behavior of the MARX generator.

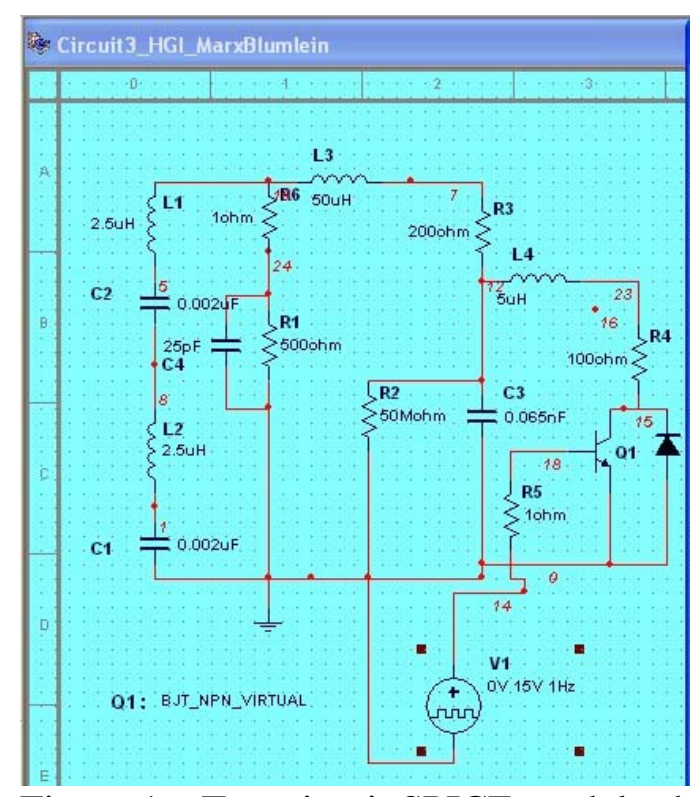

Figure 1a: Test circuit SPICE model schematic

This work was performed under the auspices of the U.S. Department of Energy by University of California, Lawrence Livermore National Laboratory under Contract W-7405-Eng-48 


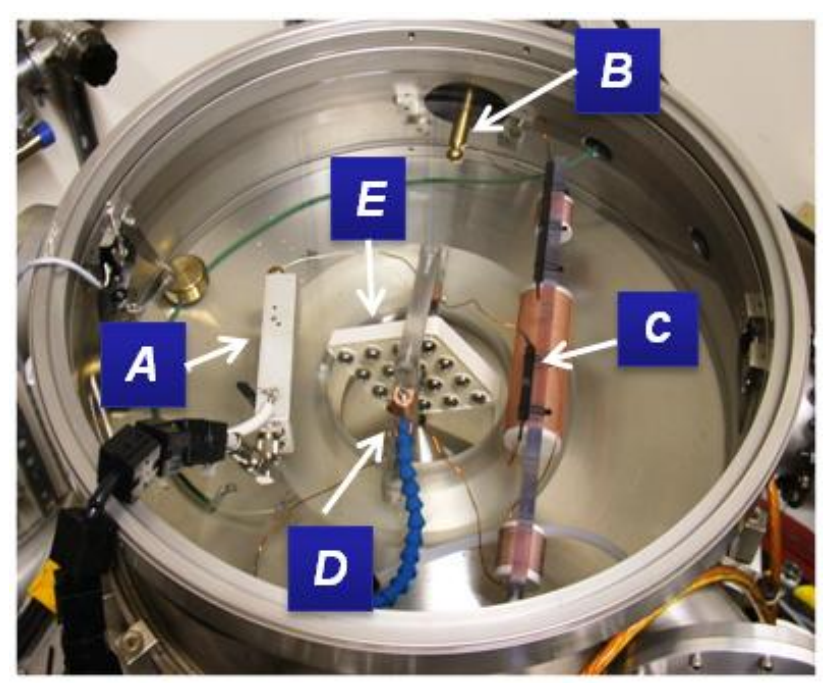

Figure 1b: Corresponding photograph.

The main components are the voltage monitor (A), the input from the MARX generator(B), the pulse shaping inductors and resistors (C) the switch(D) and the sample to be tested(E). As discussed in the introduction, the preferred testing for the insulator samples requires a voltage waveform similar to what is expected during operation. This establishes that the voltage waveform should have a charge time peaking at several hundred nanoseconds immediately followed by a high frequency oscillatory voltage. The voltage source for testing the samples was a multi-stage MARX generator (not shown) having a voltage rise-time on the order of 20ns followed by a RC decay time. To generate the appropriate test waveform, waves-shaping components were needed. A load resistor (R3) was added across the MARX output to establish a RC time constant on the order of 500ns. Also connected in parallel with the MARX was a series combination of a large inductor (L3), resistor( R3), and the insulator test sample (essentially a small capacitance (C3) as compared to the MARX equivalent capacitance(C4)). The inductor/resistor combination served multiple purposes: it isolated the MARX from the test sample in the event of a fault thereby limiting voltage reversal on the MARX capacitors; it slowed down the rise-time of the charge voltage across the sample; and it allowed the sample voltage to resonantly charge to a voltage exceeding the MARX output.

To generate the required ringing voltage, another circuit was placed in parallel with the sample. This circuit consisted of a series combination of small inductance, resistance, and switch. In the SPICE simulation, a transistor (Q1) was used to simulate the spark gap switch used in the actual circuit ( $\mathrm{D}$ in photo). The spark gap was designed to break down at the voltage peak across the test sample at which time the circuit rings with a frequency of oscillation set by the sample's capacitance and the parallel inductance with damping as determined by the resistor.

The ball-to-ball sample configuration shown in Figure 1 is shown schematically in Figure 2a where the result of an electric field modeling calculation is presented. Figure $2 b$.

$$
\begin{aligned}
& \text { Gap }=.08 ”(2.03 \mathrm{~mm}) \\
& \text { Voltage }(\mathrm{V})=224 \mathrm{kV} \\
& \text { Voltage } / \text { Gap }=110 \mathrm{kV} / \mathrm{cm} \\
& K=10
\end{aligned}
$$

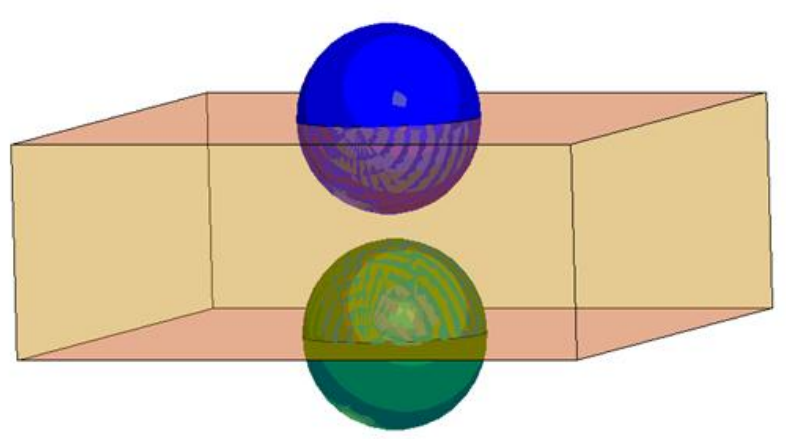

Figure 2a: Ball test schematic for field calculation. 


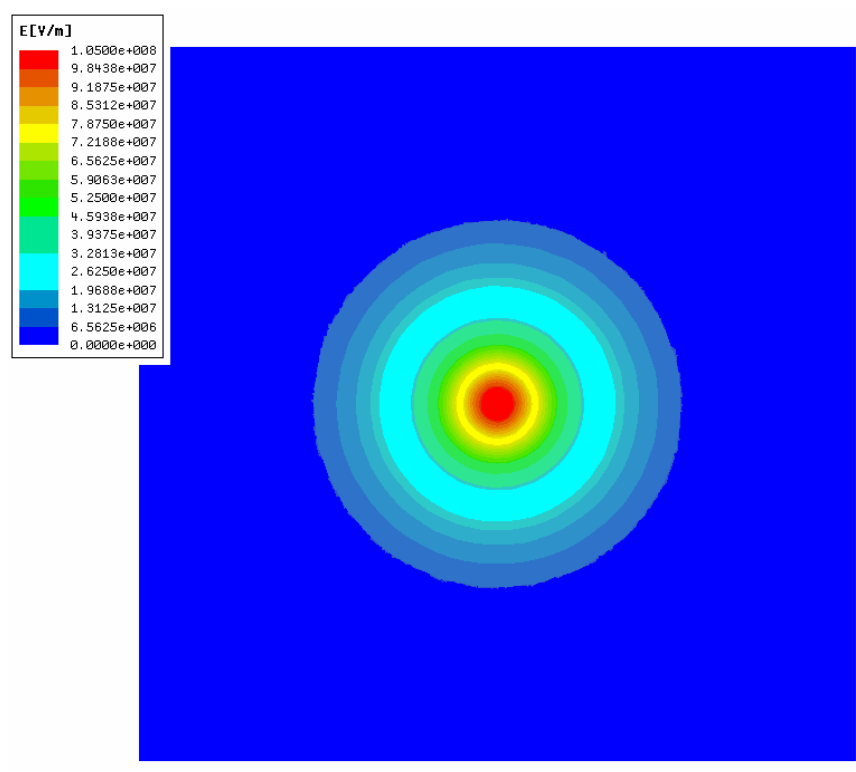

Figure 2b: Calculated fields.

It can be concluded that the electric field enhancement resulting from this particular geometry is a relatively small value of $9 \%$ making it useful for establishing the electrical characteristics of the material and not dominated by such field enhancements. An additional advantage of the ball-to-ball electrode geometry was the ability to prepare multiple samples of the dielectric under nearly identical conditions to allow the statistical characteristics of breakdown to be established. (The relatively small test area will require subsequent testing of larger area samples once the general breakdown behavior of a particular material is established). Figure 3 shows the characteristic waveforms before, during, and after breakdown that were obtained.

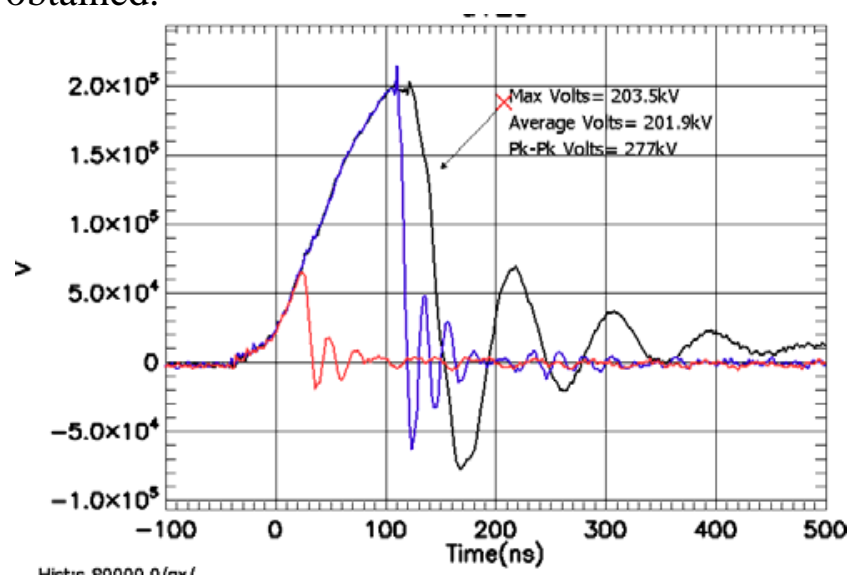

Figure 3: Scope traces of voltage monitor.
A computer-based data collection system allowed multiple pulses to be applied to each test sample until breakdown was detected. Each waveform was compared with the preceding waveform before executing the next shot. If a difference between waveforms exceeded preestablished limits, the system alerted the experimenter with a radio page. The maximum, minimum and peak-to-peak values were collected in a table along with all of the traces for subsequent inspection.

\section{RESULTS:}

The first observation concerning breakdown behavior can be made from the oscilloscope trace shown in Figure 3 since it is representative of all observed failures. In all cases the failure occurred at the positive maximum in the trace rather than during the voltage reversal. This suggests that the voltage reversal was not cause of insulator failure, at least with the extent of reversal that we were able to achieve with the test setup used in this study.

The results of testing 12 samples with a nominal $2 \mathrm{~mm}$ gap are given in Figure 4.

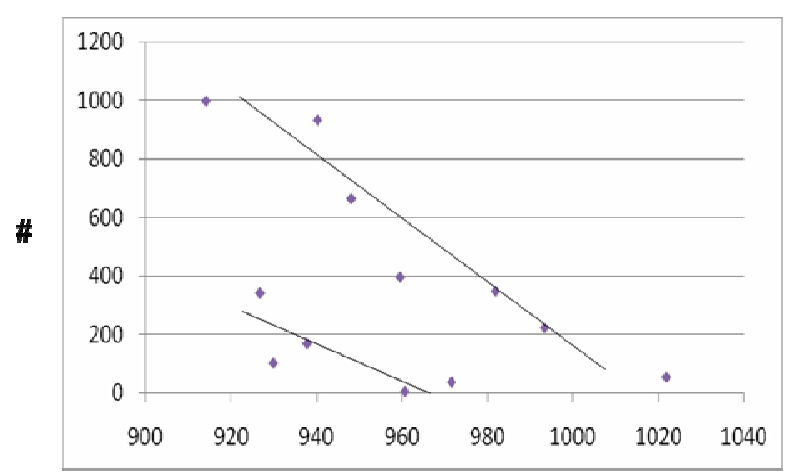

Fledd (KMem

Figure 4: Number of shots held vs. applied field for $2 \mathrm{~mm}$ gap.

The vertical axis gives the number of shots that a particular sample was able to hold vs. the electric field calculated by dividing the maximum voltage during a shot divided by the gap between the ball electrodes. (The relatively small field enhancement is ignored at this point 
since it does not change from sample to sample). As expected, the number of shots that can be held increases as the electric field decreases. The apparent bimodal character of the data suggests that there may be defect sites in the material. (The existence and characterization of such sites will be the subject of future work should we determine that this material is a suitable candidate for DWA construction). The statistical analysis of the data was done using software that came with a book on Weibull Analysis[1]. Figure 5a shows Weibull hazard plots for 1 and $2 \mathrm{~mm}$ electrode spacing at electric fields around $1 \mathrm{MV} / \mathrm{cm}$.

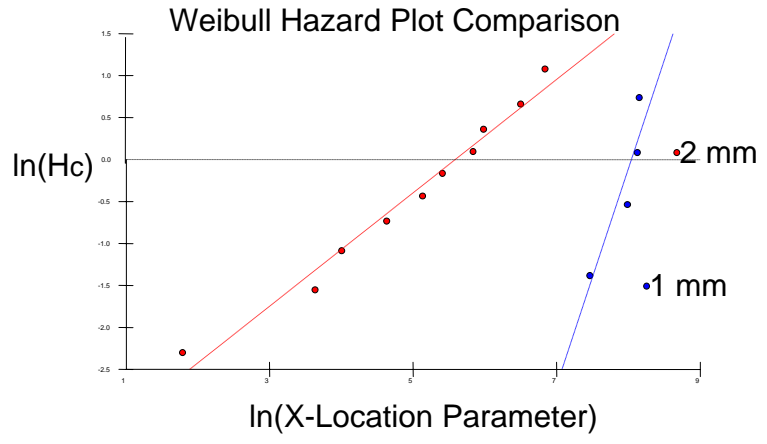

Figure 5a: Accumlated hazard plots.

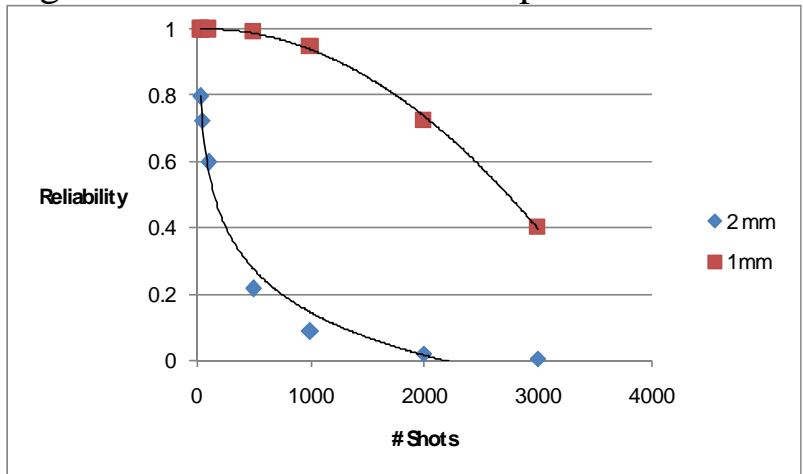

Figure 5b: Relability plots

Clearly the material under consideration will not meet our stated goal for reliability with these particular operational conditions.

To explore conditions where the material might be expected to perform better, we considered smaller electrode gaps. It is known that materials in general are able to withstand greater electric fields if they are thinner[2]. Figure 6 shows the effect of electrode spacing on breakdown field.

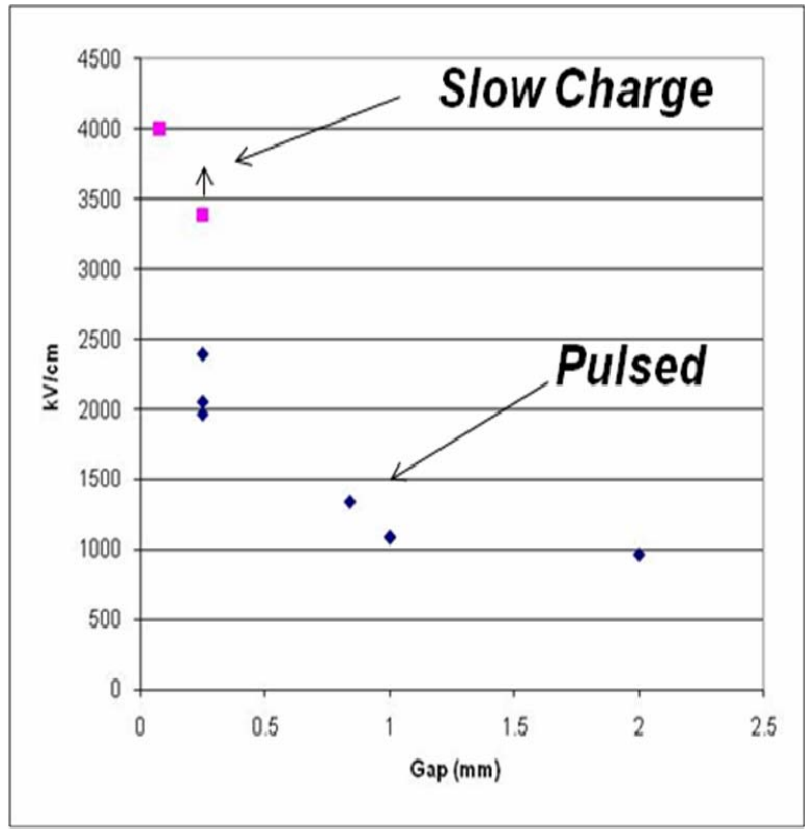

Figure 6: Effect of gap on breakdown strength.

It is clear that the reducing the electrode spacing helps to improve voltage holding performance. For instance, with a $0.25 \mathrm{~mm}$ spacing under pulse charge conditions, the breakdown field is double that found with $1 \mathrm{~mm}$ spacing, In addition to considering electrode spacing, we also investigated the effect of charging the dielectric more slowly. Since charging systems can be less expensive if the system being charged can be charged more slowly, we wanted to know if our candidate material could withstand high electric fields for longer periods of time. To make this measurement we programmed a high voltage DC power supply to ramp to full voltage in 1 second. The full voltage value was increased in $5 \mathrm{kV}$ increments. In our particular test the maximum voltage capability of our programmable power supply $(100 \mathrm{kV})$ was reached and we were not able to break our insulator material with a $0.25 \mathrm{~mm}$ gap. We were able to break the same material with a smaller $.076 \mathrm{~mm}$ gap at a lower voltage. These two slower charge measurements are also included in Figure 6 for comparison with the faster charging results. The voltage holding ability is seen to be increased by a further when a $0.25 \mathrm{~mm}$ gap was charged at a 1 second rate 
compared to charging at a 100ns rate. The arrow on the plot indicates that the sample did not break due to limitations with our programmable DC power supply. In contrast to the behavior of dielectric fluids where slower charging normally leads to diminished voltage holding capability, in the case of dielectric solids the opposite is frequently found to be the case[3,4]. Apparently this was the case for our particular material.

\section{SUMMARY AND PLANNED FUTURE WORK}

We have measured the dielectric breakdown performance of a nanoparticle composite material with a dielectric constant $=10$. Our ultimate goal is to determine if a set of operational conditions exist were this material might provide sufficient breakdown strength to be considered as a candidate material for construction of Dielectric Wall Accelerators. At this point it appears that if such conditions exist, they will involve the use of electrode spacing values around $0.25 \mathrm{~mm}$.

While it is clear that the slower charging does not adversely affect breakdown performance and might in fact improve it, further measurements will be required to quantify the effect of charging rate on breakdown performance.

Our next set of experiments will involve establishing the reliability curves for this material with the electrode spacing around 0.25 $\mathrm{mm}$ to determine if there is sufficient performance to justify further testing with larger areas.

\section{REFERENCES}

1. Dodson, The Weibull Analysis Handbook. 2nd ed. 2006, Milwaukee: American Society for Quality, Quality Press.
2. Martin, J.C., J.C. Martin on Pulsed Power. Advances in Pulsed Power Technology, ed. T.H. Martin, G. A.H., and M. Kristiansen. 1996, New York: Plenum Press. pp. 227-253.

3. Dissado, L.A. and J.C. Fothergill, Electrical Degradation and Breakdown in Polymers. IEE Materials and Devices Series 9, ed. G.C. Stevens. 1992, London, United Kingdom: Peter Peregrinus Ltd.

4. Kwan, C.K., Dielectric Phenomena in Solids with Emphasis on Physical Concepts of Electronic Processes. 2004, New York: Elsevier. 